\title{
Analysis of the cervical spine alignment following laminoplasty and laminectomy
}

\author{
Shunji Matsunaga ${ }^{1}$, Takashi Sakou ${ }^{1}$ and Kenji Nakanisi ${ }^{2}$ \\ ${ }^{1}$ Department of Orthopaedic Surgery, Faculty of Medicine, Kagoshima University; ${ }^{2}$ Department of Mechanical \\ Engineering, Faculty of Engineering, Kagoshima University, Sakuragaoka, Kagoshima, Japan
}

\begin{abstract}
Very little detailed biomechanical examination of the alignment of the cervical spine following laminoplasty has been reported. We performed a comparative study regarding the bucklingtype alignment that follows laminoplasty and laminectomy to know the mechanical changes in the alignment of the cervical spine. Lateral images of plain roentgenograms of the cervical spine were put into a computer and examined using a program we developed for analysis of the buckling-type alignment. Sixty-four patients who underwent laminoplasty and 37 patients who underwent laminectomy were reviewed retrospectively. The subjects comprised patients with cervical spondylotic myelopathy (CSM) and those with ossification of the posterior longitudinal ligament (OPLL). The postoperative observation period was 6 years and 7 months on average after laminectomy, and 5 years and 6 months on average following laminoplasty. Development of the buckling-type alignment was found in $33 \%$ of patients following laminectomy and only $6 \%$ after laminoplasty. Development of buckling-type alignment following laminoplasty appeared markedly less than following laminectomy in both CSM and OPLL patients. These results favor laminoplasty over laminectomy from the aspect of mechanics.
\end{abstract}

Keywords: laminoplasty; laminectomy; buckling; kyphosis; swan-neck deformity

\section{Introduction}

In 1930, Eiselberg ${ }^{1}$ reported a case of postoperative kyphosis of the spine following laminectomy from the 9th thoracic vertebra to the 2nd lumbar vertebra for the treatment of intramedullary tumor. Since that time a risk of inducing postoperative deformities with wide range-laminectomy, such as kyphosis and swan-neck curvature, has frequently been indicated. ${ }^{2-6}$ The fundamental principle underlying laminoplasty devised by Hattori ${ }^{7}$ is optimum conservation of the posterior supporting elements in the cervical spine and prevention of weakening of the structural support. Successful laminoplasty must overcome the problems of laminectomy. To confirm the superiority of laminoplasty, many observations on morphology of the cervical spine have been performed. However, few detailed biomechanical examinations regarding alignment of the cervical spine have been done. Buckling is a term indicating concentrated mechanical stress to a certain portion of the material, resulting from abnormal alignment that brings with it a risk of degradation of materials. ${ }^{8}$ The alignment, which causes the buckling is called buckling-type alignment. We examined the problem

Correspondence: S Matsunaga, Department of Orthopaedic Surgery, Faculty of Medicine, Kagoshima University, 8-35-1 Sakuragaoka, Kagoshima 890, Japan of postoperative abnormal alignment from the aspect of the presence or absence of buckling-type alignment.

\section{Analytical method of buckling-type alignment}

We developed a new method for the analysis of buckling-type alignment of the spine as follows. Three neighboring cervical vertebral bodies are considered as a segment unit of the spine, and the top and bottom margins of each vertebral body are expressed as data in an $\mathrm{X}-\mathrm{Y}$ orthogonal coordinate system. Approximation of the six points of the neighboring three vertebral bodies is performed by a cubic polynomial equation, $\mathrm{Y}=\mathrm{ax}^{3}+\mathrm{bx}^{2}+\mathrm{cx}+\mathrm{d}$, using the least-squares method. ${ }^{9-11}$ From the 3 rd to the 7 th cervical vertebrae, a total of five vertebral bodies, three cubic curves are drawn on the coordinate system (Figure 1). By comparing the slopes of lines tangential to these cubic curves, it is possible to judge whether or not neighboring vertebral bodies are continuous. When the slope of any line tangential to the cubic curves is changed from positive to negative at a certain position of the cervical spine, buckling is occurring there. The absolute value obtained using a two-step differential analysis of the cubic polynomial formula indicates the degree of buckling, and the locus of buckling is depicted on the graph. In addition, lateral images of spinal roentgenograms are put into a computer and 


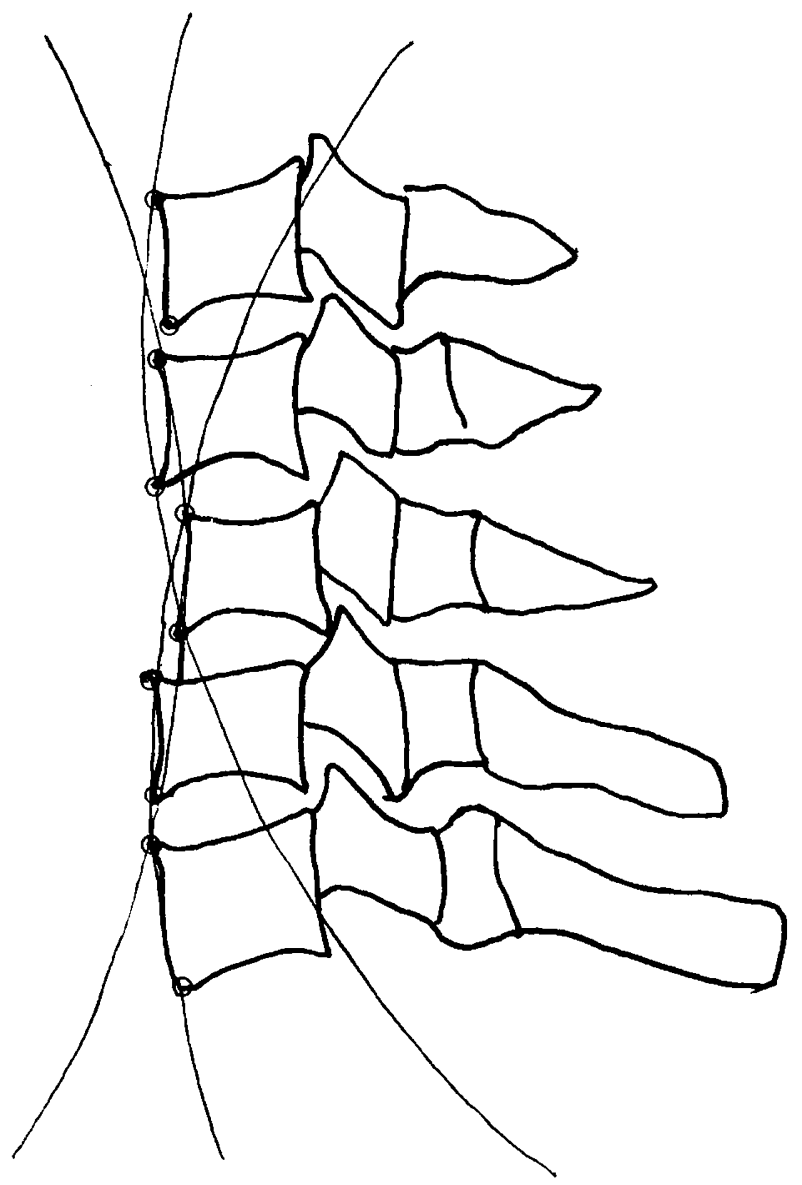

Figure 1 Buckling-type alignment. Three cubic curves are turned in different orientations. The arrow represents the locus of buckling

spinal dynamics examined using a program for analysis of the buckling alignment. Analytical results are depicted on a TV monitor. Analytical time for one subject is about $2 \mathrm{~min}$, and the analysis can be performed with an ordinary portable computer.

\section{Materials and methods}

The subjects were 64 patients ( 39 men and 25 women) who underwent laminoplasty in our institution, and 37 patients (19 men and 18 women) who underwent laminectomy in our institution during the same time period. Laminoplasty was performed according to our modified Hattori method, an alternative one-side opening method (Figure 2). After removal of postoperative drain, patients were allowed to walk wearing a conventional collar.

The subjects comprised patients with cervical spondylotic myelopathy (CSM) and those with ossification of the posterior longitudinal ligament (OPLL) but without tumor or trauma. Laminoplasty was undergone for 36 CSM patients and 28 OPLL patients. Laminectomy was undergone for $13 \mathrm{CSM}$ patients and 24 OPLL

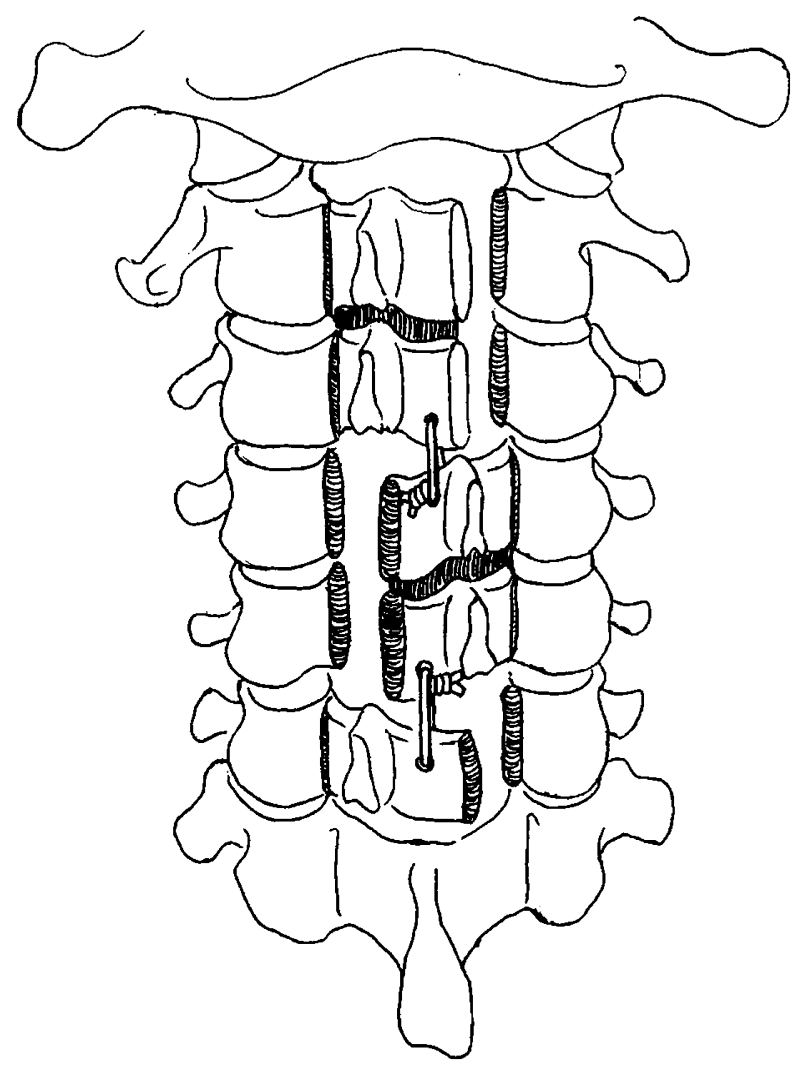

Figure 2 Schematic sketch of our method of laminoplasty

patients. Patients who underwent surgery during a certain time period were randomly allocated into two groups to avoid bias. With respect to the ossification type proposed by the Investigation Committee on Ossification of the Spinal Ligaments of the Japanese Ministry of Public Health and Welfare, ${ }^{12}$ the continuous type was found in 16 patients $(57 \%)$, the mixed type in seven patients $(25 \%)$ and the segmented type in five patients $(18 \%)$ among OPLL patients who underwent laminoplasty. As to the ossification type in those who underwent laminectomy, the continuous type was found in ten patients $(42 \%)$, the mixed type in nine patients $(38 \%)$ and the segmented type in five patients $(20 \%)$. In both surgical groups, the continuous and mixed types that exhibited wide-range ossification comprised the main portion of patients. The mean age at the time of surgery in the laminoplasty group was 59.5 years, and that in the laminectomy group was 57.1 years. Regarding the surgical range, surgery to the 3rd cervical vertebra and below was performed in 49 patients in the laminoplasty group and 26 cases in the laminectomy group. The number of patients who underwent surgery that included the 2 nd cervical vertebra was 15 in the laminoplasty group and 11 in the laminectomy group.

The patients were examined for changes in the state of curvature of the cervical spine and the presence or absence of buckling-type alignment. The postoperative observation period was 6 years and 7 months on 
average after laminectomy, and 5 years and 6 months on average after laminoplasty. The curvatures of the cervical spine were classified according to the method described by Toyama et $a l^{13}$ : curvatures were defined as the lordosis type, straight type, kyphosis type or swan-neck type based on the distance from a straight line drawn from the posterior plane of the spinal axis to the posterior plane of the 7 th cervical vertebra. As to the presence or absence of buckling-type alignment, evaluation was performed, referring to the analytical data of 100 normal healthy controls: patients demonstrating results at mean $+2 \mathrm{SD}$ and above were judged positive for the buckling-type alignment.

\section{Results}

Changes in the type of curvature of the cervical spine Before surgery, there were many lordosis type curvatures in both groups, but some patients already

Table 1 Preoperative type of curvature of cervical spine

\begin{tabular}{lccccc}
\hline & $\begin{array}{c}\text { Laminectomy } \\
(\mathrm{n}=37)\end{array}$ & \multicolumn{3}{c}{$\begin{array}{c}\text { Laminoplasty } \\
(\mathrm{n}=64)\end{array}$} \\
$\begin{array}{l}\text { Type of } \\
\text { curvature }\end{array}$ & CSM OPLL & $\begin{array}{c}\text { Type of } \\
\text { curvature }\end{array}$ & CSM OPLL \\
\hline Lordotic & 9 & 14 & Lordotic & 26 & 16 \\
Straight & 3 & 6 & Straight & 8 & 8 \\
Kyphotic & 1 & 3 & Kyphotic & 2 & 3 \\
Swan-neck & - & 1 & Swan-neck & - & 1 \\
\hline
\end{tabular}

Figures indicate the number of patients. CSM; cervical spondylotic myelopathy. OPLL; ossification of the posterior longitudinal ligament showed kyphosis or swan-neck curvatures (Table 1). The numbers of patients with lordotic or straight curvature preoperatively were 32 in the laminectomy group, and 58 in the laminoplasty group. As to the development of postoperative abnormal curvatures, five kyphosis and six swan-neck curvatures, a total of 11 of 32 cases $(34 \%)$ were found in the laminectomy group, whereas only two cases of kyphosis and two swan-neck curvatures were found in the laminoplasty group: the rate was $7 \%(4 / 58)$ or about $1 / 5$ of the rate in the laminectomy group.

Buckling analysis in 100 healthy adults

The age-matched 50 men and 50 women volunteers who had no cervical spine symptoms were analyzed. The value of buckling was $2.89 \pm 0.81 \times 10^{-4}$ $($ mean $\pm \mathrm{SD})$ in the neck flexion position, $2.67 \pm 0.91 \times 10^{-4}$ in the neutral position, and $2.59 \pm 1.01 \times 10^{-4}$ in the neck extension position. There was no significant difference in the value of buckling between men and women in any neck position.

\section{Development of buckling-type alignment}

Cases of development of buckling-type alignment after laminoplasty were markedly fewer than after laminectomy in both the CSM and OPLL patients. In particular, no buckling-type alignment was found after laminoplasty among OPLL patients (Figure 3). Buckling-type alignment developed in one $(2 \%)$ patient who underwent surgery to the 3rd cervical vertebra and below among the 49 laminoplasty patients, and in seven $(27 \%)$ among the 26 laminectomy patients.
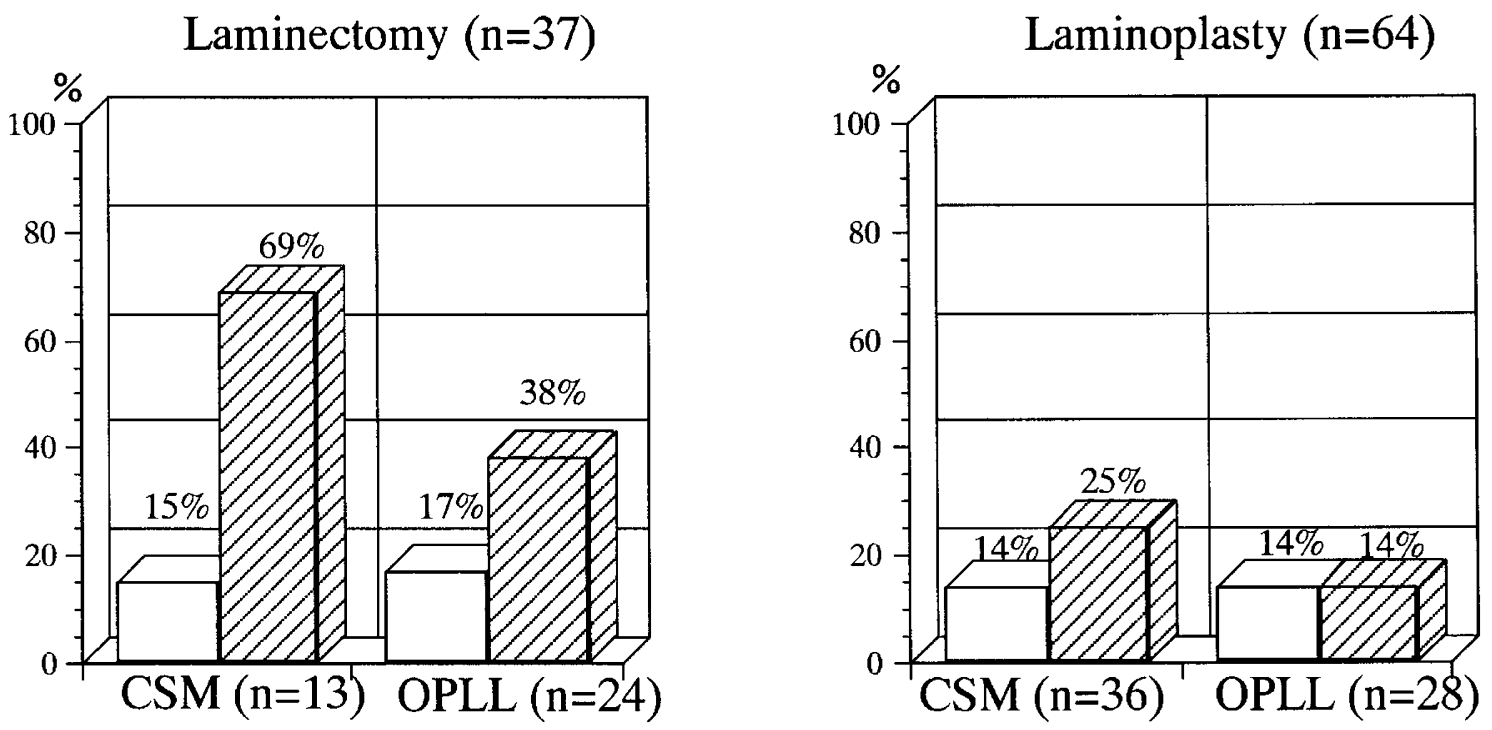

Figure 3 Rates of the development of buckling-type alignment. Patients demonstrating results at mean $+2 \mathrm{SD}$ and above were judged positive for the buckling-type alignment. White boxes represent the preoperative rates, and striped boxes represent the postoperative rates. CSM; cervical spondylotic myelopathy, OPLL; ossification of the posterior longitudinal ligament 
Buckling-type alignment developed in three (20\%) of the patients who underwent surgery including the 2 nd cervical vertebra among the 15 laminoplasty patients and six $(55 \%)$ among the 11 laminectomy patients.

Time-course of development of buckling-type alignment The development of buckling-type alignment and the subsequent course in the 14 patients who showed buckling after surgery were examined. The bucklingtype alignment developed within 3 months after surgery in most patients, and was not aggravated thereafter. However, one extended buckling-type alignment (more than $10 \times 10^{-4}$ ) was aggravated with time (Figure 4).

\section{Case presentation}

Patient 1 A 58-year-old man with cervical spondylotic myelopathy showed kyphotic deformation after laminoplasty (Figure 5a,b). The cervical spine was classified as the kyphosis-type and buckling-type alignment did not develop.

a

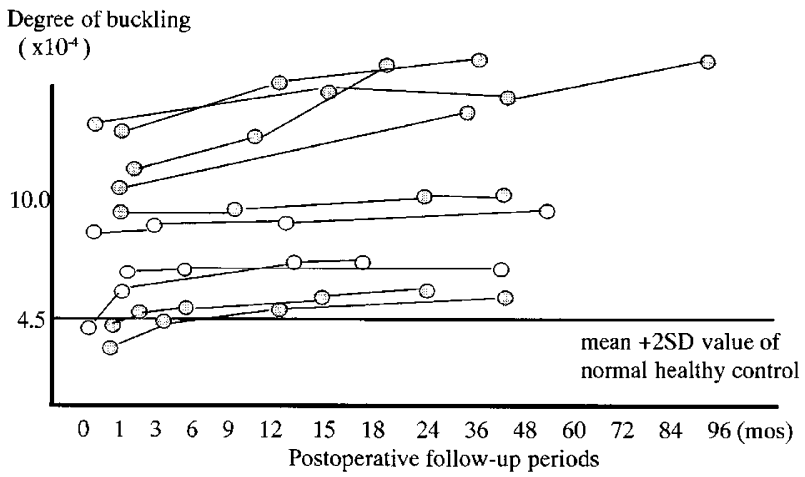

b

Degree of buckling

$\left(\mathrm{x} 10^{-4}\right)$

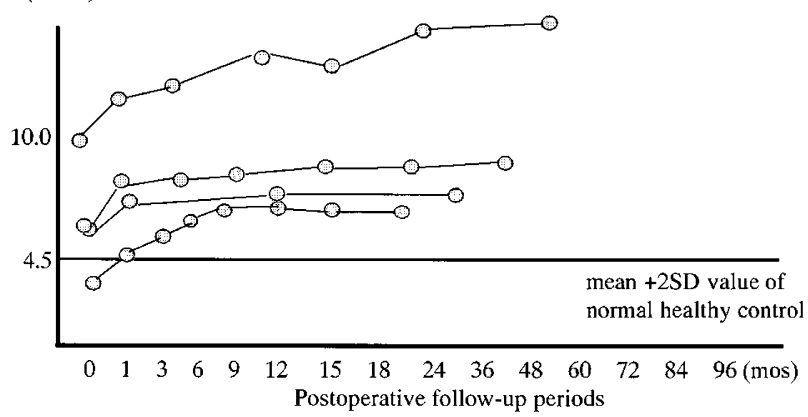

Figure 4 Time-course of development of buckling-type alignment. (a) Laminectomy group (b) Laminoplasty group. Empty circles represent patients with OPLL, and occupied circles represent patients with CSM
Patient 2 A 61-year-old man with ossification of the posterior longitudinal ligament showed the bucklingtype alignment between the 5th and 6th cervical vertebrae before surgery (Figure 6a). Nine months after laminoplasty, the buckling was not aggravated. In the buckling region, development of ossification of the anterior longitudinal ligament and progression of the OPLL occurred (Figure 6b).

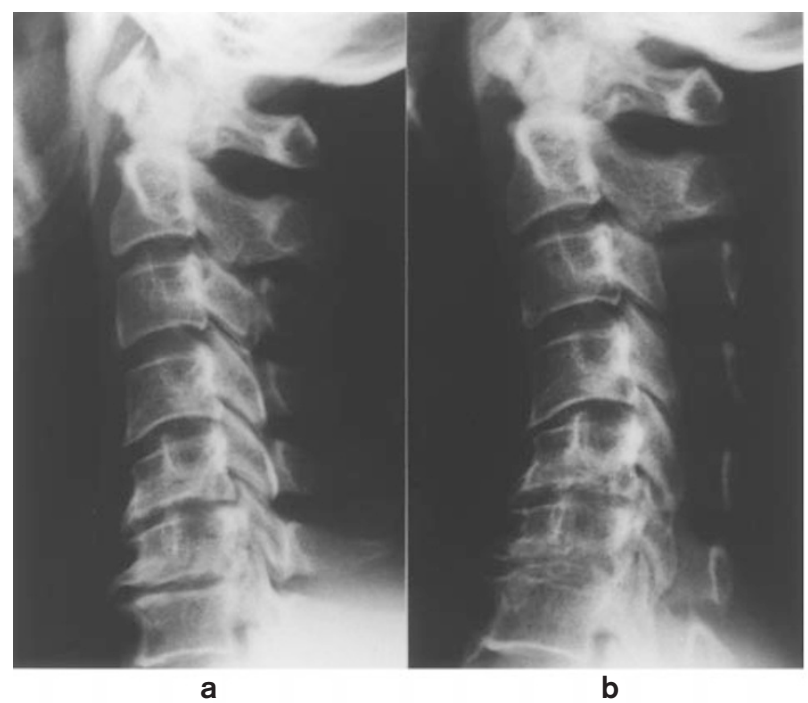

Figure 5 (a) Preoperative plain $\mathrm{X}$ ray film of cervical spine. (b) Kyphotic curvature without buckling-type alignment is seen by plain $\mathrm{X}$ ray film at 1 year after laminoplasty

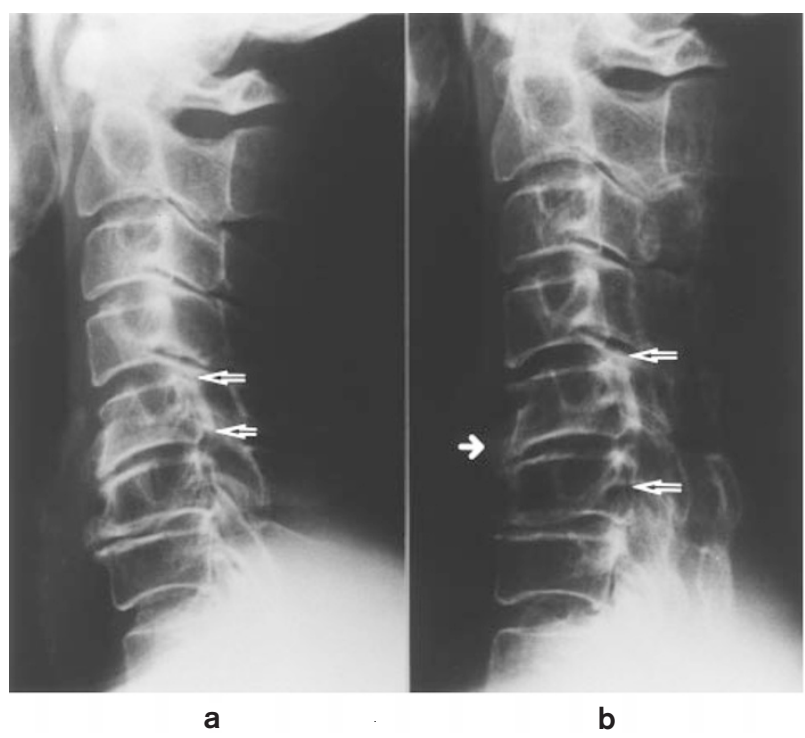

Figure 6 (a) Buckling-type alignment between the 5th and 6 th cervical vertebrae is seen before surgery. (b) Plain X ray film at 9 months after laminoplasty. Development of ossification of the anterior longitudinal ligament (right oriented arrow) and progression of the OPLL (left oriented arrows) occurred in buckling region 


\section{Discussion}

The development of abnormal curvature of the cervical spine after laminectomy has been reported by many authors. ${ }^{2-6}$ Arima examined this problem roentgenographically in detail, and reported that kyphosis or swanneck deformity occurred in $43 \%$ of patients after laminectomy of the cervical spine. ${ }^{14}$ One of the aims for developing laminoplasty was to prevent postoperative development of abnormal curvature. Various procedures for laminoplasty have been reported ${ }^{15-19}$ and the development of postoperative abnormal curvature has been examined with each procedure. ${ }^{20-24}$ According to these examinations, the incidence of postoperative abnormal curvature is about $10 \%$, although the definition and the observation period differed among investigators. According to our study, laminoplasty not only caused fewer postoperative abnormal curvatures but also induced less bucklingtype alignments than laminectomy. These results clearly indicate that from the aspect of mechanics, laminoplasty is a good procedure.

Regarding postoperative abnormal curvature, many examinations from the morphological view have been performed to date. However, quantitative evaluation of abnormal alignment is impossible with this type of examination. The method of analyzing buckling-type alignment that we have proposed is useful for the quantitative evaluation of abnormal alignments such as angular kyphosis and swan-neck deformity. From the standpoint of the construction of the cervical spine, the mechanical problem is not the mere formation of a curvature but the presence or absence of a bucking-type alignment that causes an unstable condition. Most patients with angular kyphosis and swan-neck have the buckling-type alignment.

In the patients with ossification of the posterior longitudinal ligament, postoperative development of ossification of the anterior longitudinal ligament occurred in the portion of the spine where bucklingtype alignment was present before surgery. This suggests that a mechanical factor, the buckling-type alignment, can affect the stabilizing reaction of the spine. It is of note that on subaxial subluxation after occipito-cervical fusion in patients with rheumatoid arthritis, the region of buckling-type alignment was well correlated with that of subaxial subluxation in our study. ${ }^{11}$ Existence of buckling-type alignment is one of the important factors affecting the stability of the spine

\section{References}

1 Eiselberg A. Über eine bemerkenswerde Gestaltsveränderung der Wirbelsäule nach einer ausgedehten Laminektomie wegen Rückenmarktumors. Arch Orthop 1930; 28: $132-138$.
2 Fraser RD. Orthopaedic aspects of spinal tumor in children. $J$ Bone Joint Surg 1977; 59B: 143 - 151.

3 Herkowitz HN. A comparison of anterior cervical fusion, cervical laminectomy, and cervical laminoplasty for the surgical management of multiple level spondylotic radiculopathy. Spine 1988; 13: $774-780$

4 Lonstein JE. Postlaminectomy kyphosis. Clin Orthop 1977; 128: $93-100$.

5 Sim FH et al. Swan neck deformity following extensive cervical laminectomy. J Bone Joint Surg 1974; 56A: 564-580.

6 Tachdjian $\mathrm{M}$ et al. Orthopaedic aspect of intraspinal tumor in infants and children. J Bone Joint Surg 1965; 47A: 223 - 248.

7 Hattori S. Cervical myelopathy (in Japenese). Nippon Seikeigeka Gakkai Zasshi 1978; 52: $581-593$.

8 Timoshenko S, Young DH. Strength of materials. 4th edition. D Van Nostrand: Princeton 1962, pp 268-279.

9 Bevington PR. Least squares fit to a polynomial. In: Bevington PR (ed). Data reduction and error analysis for physical science. McGraw-Hill: New York 1969, 134-163.

10 Matsunaga $\mathrm{S}$ et al. A new biomechanical analysis of the degenerative spine. In: Takahashi HE (ed). Spinal Disorders in Growth and Aging. Springer-Verlag: Tokyo 1995, pp175182.

11 Matsunaga $\mathrm{S}$ et al. Biomechanical analysis of buckling alignment of the cervical spine. Predictive value for subaxial subluxation after occipitocervical fusion. Spine 1997; 22: 765-771.

12 Tsuyama N. Ossification of the posterior longitudinal ligament of the spine. Clin Orthop 1983; 184: $71-84$.

13 Toyama $\mathrm{Y}$ et al. Realignment of postoperative cervical kyphosis in children by vertebral remodeling. Spine 1994; 22: $2565-2570$.

14 Arima T. Change of alignment following laminectomy in cervical spine (in Japanese). Brain \& Nerve Injury 1969; 1: 71-78.

15 Hirabayashi $\mathrm{K}$ et al. Extensive open-door laminoplasty for cervical stenotic myelopathy. Spine 1983; 8: 693-699.

16 Hoshino Y et al. Long term results of double door laminoplasty by longitudinal splitting of spinous process (in Japanese). Rinsho Seikei Geka 1992; 27: 257-262.

17 Nakano $\mathrm{N}$ et al. Comparison of the results of laminectomy and open-door laminoplasty for cervical spondylotic myeloradiculopathy and ossification of the posterior longitudinal ligament. Spine 1988; 13: $792-794$

18 Tomita $\mathrm{K}$ et al. Cervical laminoplasty to enlarge the spinal canal in multilevel ossification of the posterior longitudinal ligament with myelopathy. Arch Orthop Trauma Surg 1988; 107: $148-153$.

19 Tsuji H. Laminoplasty for patients with compressive myelopathy due to so-called spinal canal stenosis in cervical and thoracic regions. Spine 1982; 7: 28-34.

20 Kawakami M et al. Radiological change of the sagittal alignment and the paravertebral muscle after cervical laminoplasty (in Japanese). Seikei Geka 1993; 44: 255-260.

21 Nishi Y et al. Long-term results of extensive open-door laminoplasty (in Japanese). Rinsho Seikei Geka 1992; 27: 263 270.

22 Shimamura $\mathrm{T}$ et al. Clinical results and difficulties of laminoplastic spinal canal enlargement by sagittal splitting of spinous process for cervical myelopathy (in Japanese). Seikeigeka 1989; 40: $269-275$.

23 Sunago K et al. Postoperative radiographic evaluation of cervical laminoplasty (in Japanese). Rinsho Seikei Geka 1989; 24: 445452.

24 Yasuoka $\mathrm{S}$ et al. Incidence of spinal column deformity after laminectomy in children and adults. J Neurosurg 1982; 57: $441-$ 445. 